\title{
Microscopy techniques for dye distribution in DSCs nanocrystalline TiO2 films
}

\author{
M. A. Barreiros ${ }^{*}$, S. Sequeira*, L. C. Alves ${ }^{* *, * * *}$, V. Corregidor ${ }^{* * *, * * * * *}$, F. Guimarães ${ }^{* * * * *}$, J. Mascarenhas ${ }^{*}$, M. \\ J. Brites* \\ * Laboratório Nacional de Energia e Geologia, LEN/UES, Estrada do Paço do Lumiar, 22, 1649-038 Lisboa, \\ Portugal. \\ *** Campus Tecnológico e Nuclear, IST/ITN, Instituto Superior Técnico, Universidade Lisboa, E.N. 10, 2686- \\ 953 Sacavém, Portugal \\ **** CFNUL, Universidade de Lisboa, Av. Prof. Gama Pinto, 2, 1649-003 Lisboa, Portugal \\ ***** IPFN, Instituto Superior Técnico, Universidade de Lisboa, E.N. 10, 2686-953 Sacavém, Portugal \\ ${ }^{* * * * *}$ Laboratório Nacional de Energia e Geologia, LGM/UCTM, Rua da Amieira, Apartado 1089, 4466-901 \\ S. Mamede de Infesta, Portugal \\ email: alexandra.barreiros@lneg.pt
}

Capture of sunlight has attracted an increasing interest in the scientific community and triggered the development of efficient and cheap photovoltaic devices. Amongst recent generation technologies for solar energy conversion, dye-sensitized solar cells (DSCs) show an optimal trade-off between high-conversion efficiency and low-cost manufacturing.

For the last two decades, significant progress has been made and best energy conversion efficiency of the DSC at the laboratory scale has surpassed 12\% [1]. A lot of work has focused on the enlargement of surface areas to enhance the amount of adsorbed dyes by reduction of nanoparticle sizes or utilization of novel structures. Nevertheless there remain some crucial details of DSC operation for which limited information is available, namely dye diffusion and adsorption, surface coverage and dye distribution throughout the $\mathrm{nc}^{-\mathrm{TiO}_{2}}$ film.

Microprobe techniques can be powerful tools to evaluate the dye load, the dye distribution and dye depth profile in sensitized films. Electron Probe Microanalysis (EPMA) and Ion Beam Analytical (IBA) techniques using a micro-ion beam, namely micro-Particle Induced X-ray Emission ( $\mu$ PIXE) and Rutherford Backscattering Spectrometry (RBS), were used to quantify and to study the distribution of the ruthenium organometallic (N719) dye in $\mathrm{TiO}_{2}$ films, profiting from the different penetration depth and beam sizes of each technique. Two different types of films were prepared and sensitized, mesoporous nanoparticles and 1D nanostructured $\mathrm{TiO}_{2}$ films (figure 1).

Despite the low concentration of $\mathrm{Ru}$, the high sensitive analytical techniques used allowed to assess the $\mathrm{Ru}$ surface distribution and depth profile. Fig. 2 shows the $\mu$ PIXE maps of Ru and Ti indicating an homogeneous surface distribution. The same figure presents the RBS spectra obtained with a $2 \mathrm{MeV}$ proton beam of the same sample showing that a good spectra fit is obtained considering only two sample layers: the first one with a $1.7 \mu \mathrm{m}$ thickness; the second one being the $\mathrm{SiO}_{2}$ substrate. The $\mathrm{Ru} \mathrm{RBS}$ signal also shows that the dye has an homogeneous depth distribution.

Due to the fine spatial resolution of the EPMA/WDS (Wavelength Dispersive Spectroscopy) technique it was possible to visualise the dye distribution in sample cross-section (with micrometer or submicrometer dimensions) as presented in Fig. 3 for the elemental mapping of a mesoporous nanoparticle $\mathrm{TiO}_{2}$ film.

Dye load evaluation by two different techniques ( $\mu$ PIXE and EPMA/WDS) provided similar results $(\mathrm{Ru} / \mathrm{Ti}$ values around $0.5 \%$ ).

The distribution analysis of the organometallic dye (N719) was done through ruthenium distribution via X-ray mapping. RBS was used to assess the ruthenium depth profile. This assessment can lead to a better understanding of the device performance. 
[1] A. Yella et al., Science 334, 629-634, 2011.
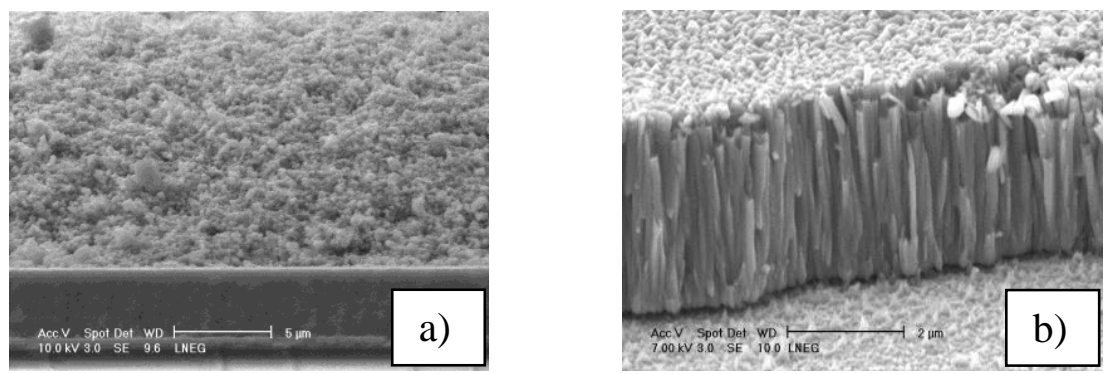

Fig. 1 - SEM (PHILIPS XL 30 FEG) image of a) mesoporous nanoparticles $\mathrm{TiO}_{2}$ film and b) $1 \mathrm{D}$ nanostructured $\mathrm{TiO}_{2}$ film.
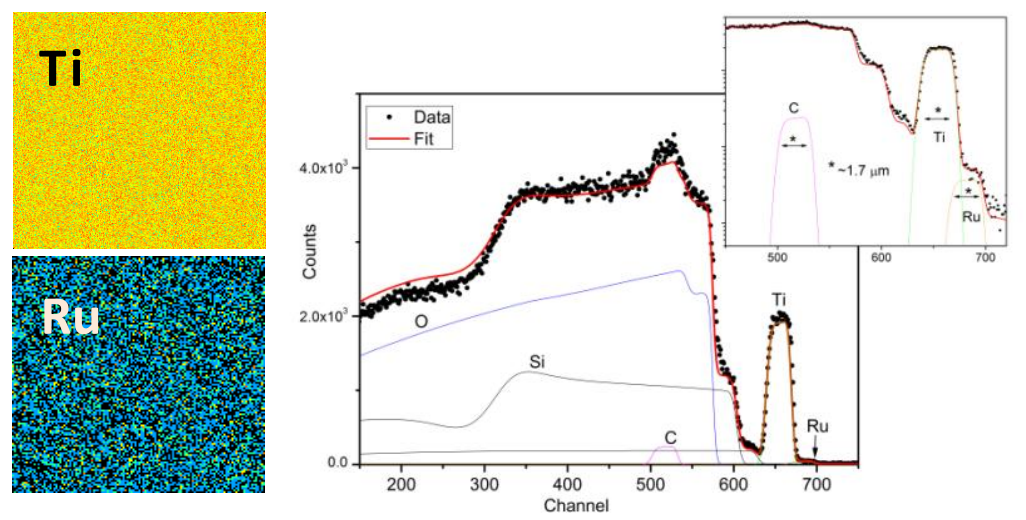

Fig. 2 - 2D $\mu$ PIXE maps for Ru and Ti (scan size: 1320x1320 $\mu \mathrm{m}^{2}$ ) and the RBS spectra recorded with a $2 \mathrm{MeV}$ alpha particle beam, both using an OM150 Oxford Microbeams setup.

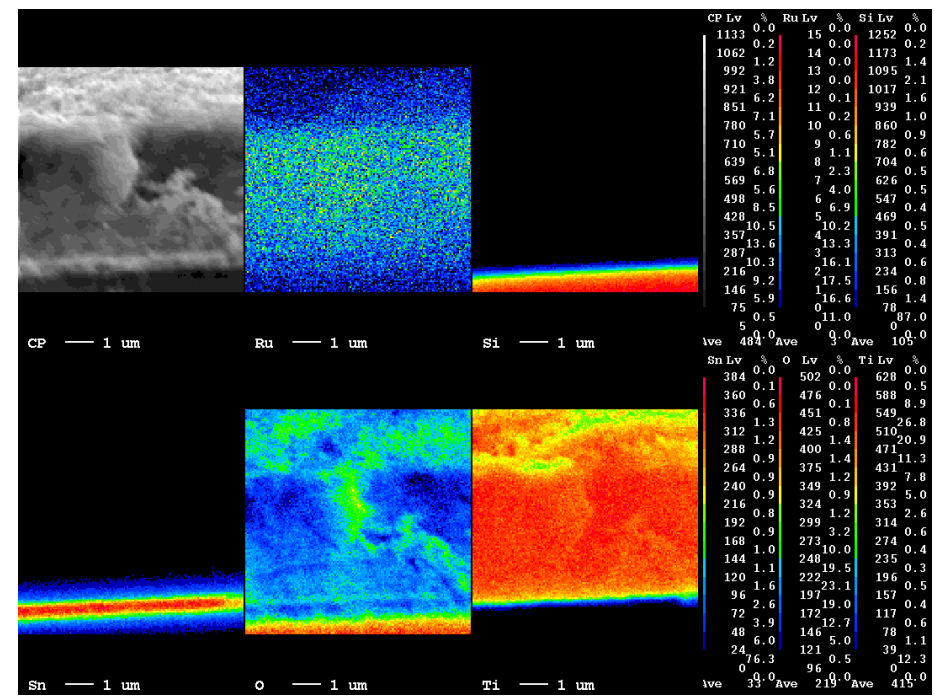

Figure 3. EPMA/WDS (JEOL JXA - 8500 F) mapping of a mesoporous nanoparticles $\mathrm{TiO}_{2}$ film. 